\title{
Coercivities of hot-deformed magnets processed from amorphous and nanocrystalline
} precursors

\author{
Xin Tang, ${ }^{\mathrm{a}, \mathrm{b}}$ H. Sepehri-Amin, ${ }^{\mathrm{a}}$ T. Ohkubo, ${ }^{\mathrm{a}}$ K. Hioki ${ }^{\mathrm{c}}$, A. Hattoric ${ }^{\mathrm{c}}$ and K. Hono ${ }^{\mathrm{a}, \mathrm{b}, ~ *}$ \\ ${ }^{a}$ Elements Strategy Initiative Center for Magnetic Materials, National Institute for Materials \\ Science, Tsukuba 305-0047, Japan \\ ${ }^{b}$ Graduate School of Pure and Applied Sciences, University of Tsukuba, Tsukuba 305-8577, \\ Japan \\ ${ }^{\mathrm{c}}$ Daido Corporate Research \& Development Center, Daido Steel Co., Ltd., Nagoya 457-8545, \\ Japan
}

\begin{abstract}
Hot-deformed magnets have been processed from amorphous and nanocrystalline precursors and their hard magnetic properties and microstructures have been investigated in order to explore the optimum process route. The hot-deformed magnets processed from an amorphous precursor exhibited the coercivity of $1.40 \mathrm{~T}$ that is higher than that processed from nanocrystalline powder, $\sim 1.28 \mathrm{~T}$. The average grain size was larger in the magnets processed from amorphous precursor. Detailed microstructure analyses by aberration corrected scanning transmission electron microscopy revealed that the $\mathrm{Nd}+\mathrm{Pr}$ concentrations in the intergranular phases were higher in the hot-deformed magnet processed from the amorphous precursor, which is considered to lead to the enhanced coercivity due to a stronger pinning force against magnetic domain wall motion.
\end{abstract}

\footnotetext{
*Corresponding author: kazuhiro.hono@nims.go.jp (K. Hono).
} 


\section{Introduction}

Optimization of hard magnetic properties of Nd-Fe-B based magnets has received considerable interest since their discovery in $1984[1,2]$. The typical coercivity of Nd-Fe-B sintered magnets with high remanence of $\sim 1.4 \mathrm{~T}$ is about $1.2 \mathrm{~T}$, which is not sufficient for the applications such as traction motors for (hybrid) electric vehicles and wind turbines. To suppress the demagnetization during service at elevated temperatures around $180^{\circ} \mathrm{C}$, the coercivity of $\mathrm{Nd}-\mathrm{Fe}-\mathrm{B}$ sintered magnets must be around $\sim 3.0 \mathrm{~T}$ at room temperature. The partial substitution of $\mathrm{Nd}$ with Dy in the $\mathrm{Nd}_{2} \mathrm{Fe}_{14} \mathrm{~B}$ main phase enhances coercivity to meet this requirement due to the higher anisotropy field of $\left(\mathrm{Nd}_{1-x} \mathrm{Dy} x\right)_{2} \mathrm{Fe}_{14} \mathrm{~B}$ [3]. However, the Dy substitution results in deterioration in magnetization due to the anti-ferromagnetic coupling between Dy and Fe. Moreover, Dy is scarce natural resources and its cost has risen because of increasing demand for high coercivity permanent magnet applications. Therefore, the development of Dy-free high-coercivity Nd-Fe-B magnets is of great industrial interests.

Lee et al. reported that the hot-deformation of melt-spun Nd-Fe-B ribbon is an effective alternative process to produce anisotropic $\mathrm{Nd}-\mathrm{Fe}-\mathrm{B}$ magnets with ultrafine-grained structure $[4,5]$. Generally, the grain size in hot-deformed magnets $(\sim 400 \mathrm{~nm})$ is one order of magnitude smaller than that of sintered magnets, which is comparable with the single domain size of the $\mathrm{Nd}_{2} \mathrm{Fe}_{14} \mathrm{~B}$ phase. Hence, the hot-deformed magnets have greater potential to achieve higher coercivity as compared with sintered magnets. This makes hot-deformed magnets as a competent candidate for the development of Dy-free high-coercivity permanent magnets. According to the grain size dependence of the coercivity of sintered magnets [6], the coercivity of the ultrafine grained magnets like hot-deformed magnets is expected to be as high as $\sim 2.5 \mathrm{~T}$; however, experimentally reported values $(\sim 1.5 \mathrm{~T})$ are much lower than the extrapolated values $[7,8]$. Based on the quantitative atom probe tomography (APT), Liu et al. 
attributed the disappointingly low coercivity of hot-deformed magnets to the intergrain exchange coupling through ferromagnetic intergranular phase [7]. In order to weaken the intergrain exchange coupling, low melting temperature RE-TM eutectic alloys were infiltrated into grain boundaries [8-11], where RE is the light rare earth elements and TM is the transition metal, by which the coercivity of $\sim 2.4 \mathrm{~T}$ was achieved without using Dy [11]. However, the remanent magnetization was degraded from $\sim 1.4 \mathrm{~T}$ to somewhat around $\sim 1.1 \mathrm{~T}$ because of the increased volume fraction of non-magnetic intergranular phases and deteriorated texture. To minimize the loss of the remanent magnetization, Akiya et al. [12] reported that the degradation of remanence can be minimized by constraining the expansion during the eutectic diffusion process and the remanence of $\sim 1.35 \mathrm{~T}$ was kept for the coercivity of 1.9 T. More recent investigations using various eutectic alloys have shown that the highest coercivity that can be achieved with the eutectic diffusion process without using Dy is $\sim 2.5 \mathrm{~T}$ [13]. The detailed microstructure analysis indicated the intergranular phase parallel to the side planes of the platelets are ferromagnetic with the amorphous structure while that along the broad surface of the platelets becomes non-ferromagnetic after the infiltration of the eutectic alloys [8]. Due to the ferromagnetism of the intergranular phases of the side planes, the pinning force against the domain wall motion is not strong enough, keeping a room for further enhancement of coercivity by microstructure control. This was also confirmed by micromagnetic simulations [8].

The microstructures of hot-deformed magnets such as grain size, their aspect ratios, chemical composition of the intergranular phases vary depending on processing conditions. Liu et al. [14] reported that the high coercivity of $\sim 1.9 \mathrm{~T}$ in ultrafine-grained $(\sim 200 \mathrm{~nm})$ hot-deformed magnets that were processed at a relatively low temperature of $750^{\circ} \mathrm{C}$. They attributed the high coercivity to both the grain size and the chemical composition of the 
intergranular phase. Note that all the hot-deformed magnets reported so far were processed from melt-spun nanocrystalline precursors. By increasing the cooling rate during melt-spinning, it is possible to prepare amorphous powders. In this work, we investigated magnetic properties and microstructures of the hot-deformed magnets that were manufactured from amorphous and nanocrystalline precursors to explore optimum processing routes to maximize the coercivity.

\section{Experimental procedures}

The alloy ingots with the nominal composition of $\mathrm{Nd}_{28.4} \mathrm{Pr}_{0.06} \mathrm{Fe} \mathrm{bal} \mathrm{C} 03.41 \mathrm{Ga} 0.53 \mathrm{Al} \mathrm{l}_{0.06} \mathrm{~B}_{0.97}$ (wt.\%) were prepared by melting high-purity constituent elements. The alloy ingots were melt-spun at different roll speeds and then amorphous and nanocrystalline melt-spun ribbons with the same chemical composition were obtained. The as-melt-spun ribbons were crushed to small size particles. Subsequently, these powders were compacted to above $98 \%$ of full density by hot-pressing at $650{ }^{\circ} \mathrm{C}$ under $380 \mathrm{MPa}$ in vacuum. The hot-pressed magnet processed from crystalline powder (HPM-C) and that processed from amorphous powder (HPM-A) were obtained. These two kinds of compacts were subsequently hot-deformed at $850^{\circ} \mathrm{C}$ at an average speed of $5.5 \mathrm{~mm} / \mathrm{min}$ until their $75 \%$ height reduction were achieved. The hot-deformed magnets processed from the crystalline powder (HDM-C) and amorphous powder (HDM-A) with full density were fabricated. The overall structures of the samples were identified by X-ray diffraction (XRD) with $\mathrm{Cu} K_{\alpha}$ radiation. The magnetic properties of the powders, hot-pressed and hot-deformed samples were measured using a superconducting quantum interface device vibrating sample magnetometer (SQUID-VSM) and a BH tracer after magnetization in a pulsed magnetic field of $7 \mathrm{~T}$. The microstructures were investigated using a scanning electron microscope (SEM), Carl Zeiss CrossBeam1540EsB, and a scanning 
transmission electron microscope (STEM) using a Titan $\mathrm{G}^{2}$ 80-200 system with a probe aberration corrector.

\section{Results}

\subsection{XRD patterns and magnetic properties}

Fig. 1 shows the XRD patterns for melt-spun powders, hot-pressed magnets and hot-deformed magnets. No recognizable characteristic peaks are observed in the XRD pattern of the amorphous powder. In contrast, distinct crystalline peaks from the 2:14:1 phase are observed in the nanocrystalline powders. After hot-pressing at $650^{\circ} \mathrm{C}$, the amorphous phase is crystallized and the sharp crystalline peaks from the $2: 14: 1$ phase can be recognized in the XRD profile from the HPM-A sample. This indicates that the amorphous phase is crystallized during the hot-pressing process. As a consequence, the XRD pattern for HPM-A is similar to that for HPM-C and both XRD results show that the orientations of grains are random in the hot-pressed stage (MQ2). After hot-deformation with the $75 \%$ height reduction, the relative intensities of the diffraction peaks of (004), (105), (006) and (008) increased dramatically, suggesting that a crystallographic alignment has developed in hot-deformed magnets (MQ3).

Fig. 2 shows magnetic hysteresis loops for the initial amorphous and nanocrystalline powders and the demagnetization curves of hot-pressed and hot-deformed magnets. As shown in Fig. 2(a), the amorphous powders show very low coercivity $\left(\mu_{0} H_{\mathrm{c}}=0.029 \mathrm{~T}\right)$ and remanent magnetization $\left(\mu_{0} M_{\mathrm{r}}=0.21 \mathrm{~T}\right)$, while nanocrystalline powders show substantially higher $\mu_{0} H_{\mathrm{c}}$ of $1.27 \mathrm{~T}$ because of the formation of the hard magnetic $2: 14: 1$ phase. The remanence of $\mu_{0} M_{\mathrm{r}}=0.85 \mathrm{~T}$ is higher than half of the saturation magnetization, $0.7 \mathrm{~T}$, suggesting the remanence enhancement effect due to the exchange coupled nanocrystals. By hot-pressing amorphous powders at $650^{\circ} \mathrm{C}$, the coercivity and remanence are enhanced substantially to $\sim 1.67 \mathrm{~T}$ and $\sim 0.8 \mathrm{~T}$, respectively, in HPM-A. The enhanced coercivity of HPM-A is ascribed to the crystallization of the $2: 14: 1$ phases during the hot-pressing process. The coercivity of 
HPM-C is $\sim 1.46 \mathrm{~T}$, which is higher than that of the nanocrystalline precursors and lower than that processed from amorphous precursors, HPM-A. After the hot-deformation at $850^{\circ} \mathrm{C}$, the remanent magnetizations of both samples were increased to $\sim 1.42 \mathrm{~T}$ because of the development of a strong (001) texture during the hot-deformation process, which is consistent with the XRD results. The coercivities for HDM-C and HDM-A were decreased to $\sim 1.28 \mathrm{~T}$ and $\sim 1.4 \mathrm{~T}$ in comparison with their hot-pressed samples because of the strong c-axis alignment of the $\mathrm{Nd}_{2} \mathrm{Fe}_{14} \mathrm{~B}$ grains [15]. It should be noted that the remanent magnetizations of the hot-deformed magnets produced from nanocrystalline and amorphous precursors are almost the same; however, the coercivity of the hot-deformed magnet processed from the amorphous precursor is higher than that processed from the nanocrystalline precursor. In order to understand the difference in the coercivities of these two hot-deformed magnets

processed from amorphous and nanocrystalline precursors, the microstructure characterizations were carried out.

\subsection{Microstructure of magnetic powders}

Fig. 3 shows the microstructure of nanocrystalline and amorphous powders. The bright field TEM image in Fig. 3(a) indicates that the average diameter of the equiaxed grains is $\sim 24.9 \mathrm{~nm}$. The selected area diffraction pattern (SADP) in the inset of Fig. 3 (a) shows ring patterns expected from randomly oriented nanocrystalline powders. The bright field TEM image of the amorphous powder is shown in Fig. 3(b), in which small nanocrystals of about $5 \mathrm{~nm}$ in diameter are embedded in the amorphous matrix. As a result, the SADP (inset figure) exhibits a typical halo ring for amorphous structure.

\subsection{Microstructure of hot-pressed magnets}


Fig. 4 shows the bright field TEM images, high resolution STEM images and compositional profiles across the grain boundaries for HPM-C and HPM-A. Equiaxed grains are observed in Figs. 4(a) and (b); the spherical morphology of the grains is similar to the conventional MQ1 powder. Note that this result is not consistent with the previous report by Liu et al. [16], in which they reported elongated grains of the 2:14:1 phases after hot-pressing at $750^{\circ} \mathrm{C}$. This discrepancy may be due to the lower temperature $\left(650^{\circ} \mathrm{C}\right)$ for hot-pressing in this work. The mean grain sizes for HPM-C and HPM-A were estimated to be $\sim 33.6 \mathrm{~nm}$ and $42.6 \mathrm{~nm}$, respectively. The presence of continuous rings from (006) planes in the inset SADPs of Figs. 4(a) and (b) indicates that the $c$-axes of grains are randomly distributed with the isotropic feature of the microstructure. High-angle annular dark-field (HAADF) images taken from grain boundaries for HPM-C and HPM-A are shown in Fig. 4(c) and Fig. 4(d), respectively. As seen in Fig. 4(c), an amorphous Nd-rich phase indicated by red arrow appears at the grain boundary in HPM-C. The width of grain boundary phase shrinks along the grain boundary and the lattice fringes from two grains become diffuse at the end of the intergranular phase as marked by dashed circle, which indicates physical contact as well as exchange coupling between the two grains. As a result, the networking of the intergranular phase becomes discontinuous. The inset superimposed EDS mapping of $\mathrm{Nd}+\mathrm{Fe}$ in Fig. 4(c) demonstrates that a slight enrichment of $\mathrm{Nd}$ in this grain boundary phase. The detailed composition profile across the grain boundary phase obtained by a line scan shows about $\sim 20$ at. $\%$ of $\mathrm{Nd}+\mathrm{Pr}$ concentration in this grain boundary phase, Fig. 4 (e). Fig. 4(d) shows a high resolution STEM-HAADF image taken from a grain boundary in HPM-A. Further compositional analysis in the grain boundary phase in Fig. 4(f) indicates that about $\sim 25$ at.\% of $\mathrm{Nd}+\mathrm{Pr}$ is enriched in this grain boundary phase, which is higher than the concentration of $\mathrm{Nd}+\mathrm{Pr}$ in the grain boundary phase of HPM-C in Fig. 4(e). The higher Nd+Pr concentration in 
the intergranular phase in HPM-A is originated from the smaller volume of grain boundaries with bigger grain size in HPM-A.

\subsection{Microstructure of hot-deformed magnets}

The overall microstructures of the hot-deformed magnets processed from the nanocrystalline and amorphous precursors are shown in Fig. 5. Figs. 5 (a) and (b) show low magnification back scattered electron (BSE) SEM images with $c$-axis in-plane for HDM-C and HDM-A, respectively. In these images, the grey contrast corresponds to $2: 14: 1$ grains while the brightly imaging lined-up grains are the neodymium oxide that were formed on the surface of ribbons $[7,8]$. The magnified images taken from the HDM-C and HDM-A samples are presented in Fig. 5(c) and (d), respectively. The platelet-shaped grains with $c$-axes parallel to applied stress direction shown by arrow (P) are formed after hot-deformation and the $\mathrm{Nd}_{2} \mathrm{Fe}_{14} \mathrm{~B}$ grains with grey contrast are separated by a thin intergranular phase with a bright contrast. Based on these two BSE SEM images, the grain size of each sample is statistically calculated. The average grain sizes of $\mathrm{Nd}_{2} \mathrm{Fe}_{14} \mathrm{~B}$ in $\mathrm{HDM}-\mathrm{C}$ are $\sim 375 \mathrm{~nm}$ along the $c$-plane and $\sim 106 \mathrm{~nm}$ in the perpendicular direction to the $c$-plane. By fabricating the sample from the amorphous precursor, the dimension along $c$-plane direction decreases to $\sim 343 \mathrm{~nm}$ while the thickness increases to $\sim 132 \mathrm{~nm}$. The average aspect ratio in HDM-A is $\sim 2.60$, which is smaller than that $(\sim 3.54)$ in HDM-C. This also can be confirmed by the BSE SEM images. Here, the grains in Fig. 5(c) show more elongated shape compared to those in Fig. 5(d). It is noteworthy that the networking of Nd-rich intergranular phase is clearer and more continuous in Fig. 5(d), especially in the side planes of the platelet-like grains [7], which will be discussed in detail later. The areal fraction of the intergranular phase in HDM-C and HDM-A is calculated to be $\sim 2.58 \%$ and $\sim 2.27 \%$, respectively, which are comparable with the value 
reported in ref. [12]. More details about the statistical comparison between HDM-C and HDM-A are summarized in Table 1.

Fig. 6 and Fig. 7 show the STEM-EDS analyses of HDM-C and HDM-A, respectively. The contrast in the HAADF images in Fig. 6(a) and Fig. 7(a) indicate that the grain boundaries are enriched with a heavier element, i.e. $\mathrm{Nd}$. This is consistent with the EDS mapping of $\mathrm{Nd}$ in Fig. 6(b) and Fig. 7(b), where $\mathrm{Nd}$ is strongly concentrated in the intergranular regions and triple junctions. Moreover, the grains in HDM-A are larger than those in HDM-C, which is also documented in Fig. 5. Since grain coarsening is always accompanied by the disappearance of grain boundaries, the volume of grain boundaries in HDM-A is expected to be smaller than that in HDM-C. Given the same Nd content in these two samples, stronger $\mathrm{Nd}$ enrichment in grain boundaries is expected in HDM-A. As a result, the networking of grain boundary phase is more continuous and obvious in HDM-A sample. As seen in Fig. 7(b) and Fig. 7(c), more continuous networking of grain boundary phases is observed in the $\mathrm{Nd}$ and Fe mappings of HDM-A. It is not surprising to find that Ga is also enriched in these triple junctions in both samples [7]. The Ga enrichment is reportedly beneficial to the coercivity due to the improved wettability of Nd-Ga-rich phase and better isolation is thus achieved between the hard magnetic grains [17-19].

Fig. 8 shows the high-resolution STEM-HAADF images taken from the $c$-plane interfaces between two $\mathrm{Nd}_{2} \mathrm{Fe}_{14} \mathrm{~B}$ grains in HDM-C and HDM-A. The compositional profiles taken across $c$-plane grain boundaries in HDM-C in Fig. 8(d-f) were obtained from superimposed EDS mappings in Figs. 8(a-c), respectively. These compositional profiles show that $\mathrm{Nd}$ is strongly enriched in the intergranular phase. The periodic compositional profiles of $\mathrm{Nd}+\mathrm{Pr}$ and $\mathrm{Fe}+\mathrm{Co}$ results from the periodical stacking of $\mathrm{RE}$ rich layers in the $\mathrm{Nd}_{2} \mathrm{Fe}_{14} \mathrm{~B}$ structure, suggesting the EDS analysis has sufficient special resolution to determine the chemical 
composition of the thin intergranular phase. The concentrations of $\mathrm{Nd}+\mathrm{Pr}$ in these three typical $c$-plane intergranular phases of HDM-C were determined to be $\sim 50.2$ at. $\%, \sim 42.5$ at.\% and $\sim 35.2$ at.\%. Accordingly, $\sim 39.6$ at. $\%, \sim 50.7$ at. $\%$ and 53.7 at. $\%$ of $\mathrm{Fe}+\mathrm{Co}$ were contained in these three grain boundary phases of HDM-C. In contrast, the compositional profiles of $c$-plane grain boundary phases in HDM-A shown in Figs. 9(g-i) obtained from the superimposed mappings in Fig. $9(\mathrm{j}-1)$ indicate $\sim 62.8$ at. $\%, \sim 50.1$ at. $\%, \sim 50.5$ at. $\%$ for $\mathrm{Nd}+\mathrm{Pr}$ and $\sim 22.5$ at.\%, $\sim 41.5$ at. $\%, \sim 41.3$ at. $\%$ for $\mathrm{Fe}+\mathrm{Co}$ were contained in these three intergranular phases, considerably richer in RE compared to those in HDM-C.

Fig. 9 presents the high-resolution STEM-HAADF images taken in the side-plane interface of two $\mathrm{Nd}_{2} \mathrm{Fe}_{14} \mathrm{~B}$ grains and EDS line compositional profiles taken across typical Nd-rich intergranular phases in HDM-C and HDM-A. In Fig. 9(a-c), thin intergranular phases are formed in side-plane grain boundary of HDM-C, which is enriched with $\mathrm{Nd}$ and depleted from Fe. In contrast, the contrasts from the intergranular phase in the side-plane grain boundary of HDM-C in Fig. 9(a-c) show weaker intensity in the $\mathrm{Nd}+\mathrm{Fe}$ mapping as compared to those in $c$-plane intergranular phases (Fig. 8(a-c)), suggesting relatively low $\mathrm{Nd}$ concentration in the side-plane intergranular phases as recently reported by Sasaki et al. [20]. The composition of the intergranular phase is qualitatively determined by the EDS compositional profiles shown in Fig. $9(\mathrm{~d}-\mathrm{f})$, indicating contents of $\mathrm{Nd}+\mathrm{Pr}$ and $\mathrm{Fe}+\mathrm{Co}$ in these three side-plane intergranular phases in HDM-C are close to $\sim 28.6$ at. $\%, \sim 29.5$ at. $\%, \sim 33.1$ at.\% and $\sim 61.9$ at. $\%, \sim 67.1$ at. $\%, \sim 56.4$ at.\%, respectively. As for the intergranular phase in the side-plane grain boundary of HDM-A, a clear contrast from an amorphous intergranular phase is observed in Fig. 9(j-1). Compared to the intergranular phases in the side-plane intergranular phases in HDM-C in Fig. 9(a-c), the grain boundary phases shown in Fig. 9(j-1) are thicker with clearer contrast, meaning relatively higher $\mathrm{Nd}+\mathrm{Pr}$ content in these intergranular phases. 
This is further confirmed by the EDS line profiles shown in Fig. $9(\mathrm{~g}-\mathrm{i})$, in which $\sim 41.3$ at.\%, $\sim 43.4$ at.\%, $\sim 35.8$ at.\% in $\mathrm{Nd}+\mathrm{Pr}$ and $\sim 52.2$ at.\%, $\sim 42.2$ at.\%, $\sim 57.4$ at.\% in $\mathrm{Fe}+\mathrm{Co}$ were measured in these typical side-plane intergranular phases in HDM-A.

To obtain the composition of intergranular phases more statistically, we observed at least five intergranular phases (not shown here) for each case. The average concentrations of $\mathrm{Nd}+\mathrm{Pr}$ and $\mathrm{Fe}+\mathrm{Co}$ for the intergranular phases in $c$-plane and side-plane in HDM-C and HDM-A are summarized in Table 2. In HDM-C, the amount of $\mathrm{Nd}+\mathrm{Pr}$ elements in the $c$-plane grain boundaries is about $\sim 39.9$ at. $\%$ and this value decreases to $\sim 28.3$ at. $\%$ in the side-plane grain boundaries. The average concentration of ferromagnetic elements $\mathrm{Fe}+\mathrm{Co}$ is $\sim 51.2$ at.\% in the $c$-plane intergranular phases and $\sim 64.4$ at. $\%$ in the side-plane intergranular phases. A considerable difference in $\mathrm{Nd}+\mathrm{Pr}$ and $\mathrm{Fe}+\mathrm{Co}$ contents between the $c$-plane and side-plane intergranular phases is observed, which is consistent with other reports $[8,15]$. It was reported in sintered magnets that $\mathrm{Nd}$ is highly segregated in the $c$-plane intergranular phases with a crystalline structure while $\mathrm{Nd}$ concentration is low in the side-grain intergranular phases with an amorphous structure [20-21]. Here, the intergranular phases in the $c$-plane and the side-plane grain boundaries in the hot-deformed magnets are all shown to be amorphous structure but much different in composition between c-plane and side-plane. As for the composition of the intergranular phases in HDM-A, the content of $\mathrm{Nd}+\mathrm{Pr}$ in the $c$-plane and side-plane grain boundaries are in average $\sim 50.1$ at. $\%$ and $\sim 34.4$ at. $\%$, respectively, while the concentrations of ferromagnetic elements $\mathrm{Fe}+\mathrm{Co}$ in the $c$-plane and side-plane intergranular phases were determined to be $\sim 40.2$ at.\% and $\sim 57.9$ at. $\%$, respectively. In comparison with the composition of intergranular phases in $\mathrm{HDM}-\mathrm{C}$, higher $\mathrm{Nd}+\mathrm{Pr}$ and lower $\mathrm{Fe}+\mathrm{Co}$ contents are found in both $c$-plane and side-plane intergranular phases in HDM-A, which resulted from the optimized aspect ratio and resultant smaller areal fraction of grain boundaries in HDM-A 
as shown in Table 1. Previous works have unequivocally demonstrated that an increase in $\mathrm{Nd}$ content and a decrease in concentration of ferromagnetic elements in the intergranular phases would result in enhanced pinning force against domain wall motion during magnetization reversal process $[7,8]$. Consequently, the coercivity of HDM-A is higher than that of HDM-C.

\section{Discussion}

The amorphous precursor shows very low coercivity because of net zero-magnetocrystalline anisotropy [22-24]. The microstructure of the amorphous powders shown in Fig. 3(b) indicates that a few nanocrystallites in $\sim 5 \mathrm{~nm}$ diameter are embedded in an amorphous matrix. The crystalline precursor (MQ1) that is commonly used for the production of hot-deformed magnets shows fine $\mathrm{Nd}_{2} \mathrm{Fe}_{14} \mathrm{~B}$ nanocrystals with an average grain size of $\sim 24.9 \mathrm{~nm}$ (Fig. 3(a)). This grain size is substantially larger than the exchange length of the $\mathrm{Nd}_{2} \mathrm{Fe}_{14} \mathrm{~B}$ phase $\left(l_{\mathrm{ex}} \sim 1.9 \mathrm{~nm}\right)$ [25]. Hence the magnetocrystalline anisotropy is no longer averaged out, and the coercivity of nanocrystalline powders becomes two orders of magnitude larger than that of the amorphous precursor as shown in Fig. 2(a). However, the remanent magnetization of the nanocrystalline power is approximately $0.6 M_{\mathrm{s}}$ (remanence enhancement), indicating the randomly oriented nanocrystals are exchange coupled.

After hot-pressing the nanocrystalline and amorphous precursors at $650{ }^{\circ} \mathrm{C}$, both of them were fully crystallized (Fig. 1); this is because the crystallization temperature of $\mathrm{Nd}_{2} \mathrm{Fe}_{14} \mathrm{~B}$ is lower than the hot-pressing temperature. The average grain size of HPM-A, $\sim 42.6 \mathrm{~nm}$, is larger than that of HPM-C, $\sim 33.6 \mathrm{~nm}$. Compared to the high number density of $\mathrm{Nd}_{2} \mathrm{Fe}_{14} \mathrm{~B}$ nanocrystallites in the nanocrystalline powders, the number density of nanocrystals in the

amorphous precursor is very small (Fig. 3 (b)). These pre-existing nanocrystals in the 
amorphous powders is considered to act as nucleation sites for crystallization during the subsequent hot-pressing process, indicating the number density of nuclei for crystallization is limited in the amorphous powder. Based on thermodynamic calculation for the crystallization of $\mathrm{Nd}_{2} \mathrm{Fe}_{14} \mathrm{~B}$ in amorphous powders, the nucleation activation energy $E_{\mathrm{n}}$ and grain growth activation energy $E_{\mathrm{g}}$ for $\mathrm{Nd}_{2} \mathrm{Fe}_{14} \mathrm{~B}$ nanocrystals were reported to be $\sim 0.9 \mathrm{eV}$ and $\sim 0.5 \mathrm{eV}$, respectively [26]. The high ratio $E_{\mathrm{n}} / E_{\mathrm{g}}>1$ indicates that the growth for $\mathrm{Nd}_{2} \mathrm{Fe}_{14} \mathrm{~B}$ is kinetically favorable as compared to the nucleation of $\mathrm{Nd}_{2} \mathrm{Fe}_{14} \mathrm{~B}$ in amorphous powders during hot-pressing process. Consequently, coarser grains are yielded in HPM-A.

Manaf et al [27] reported that the coercivity was improved from $\sim 1.2 \mathrm{~T}$ to $\sim 2.0 \mathrm{~T}$ with increasing the grain size from $\sim 15 \mathrm{~nm}$ to $\sim 50 \mathrm{~nm}$ in the over-stoichiometric melt-spun ribbons; further numerical micromagnetic analyses clearly demonstrated that stronger intergrain exchange interactions account for the deterioration in coercivity of isotropic nanocrystalline Nd-Fe-B ribbons [28]. Furthermore, smaller grain size means a larger volume fraction of grain boundaries and resultant lower $\mathrm{Nd}+\mathrm{Pr}$ concentration in the intergranular phases in HPM-C as shown in Fig. 4, which is considered to be detrimental to the coercivity. This explains the tendency of coercivity dependence on grain size in hot-pressed nanocrystalline powders; $\mu_{0} H_{\mathrm{c}}=1.27 \mathrm{~T}$ for the grain size of $\sim 24.9 \mathrm{~nm}$ in nanocrystalline powders and $\mu_{0} H_{\mathrm{c}}=1.46 \mathrm{~T}$ for the grain size of $\sim 33.6 \mathrm{~nm}$ in HPM-C as shown in Fig. 2 (b).

Concerning the coercivity in hot-deformed magnets, it is considered that magnetization reversal process takes place by the nucleation and propagation of reversed magnetic domain wall. The micromagnetic simulation results $[7,8]$ demonstrated that a ferromagnetic phase with lowered magnetocrystalline anisotropy as intergranular phase facilitates the nucleation of reversed magnetic domain. Once a reversed domain is nucleated, it can easily propagate through adjacent grains because of small pinning force against the domain wall motion. By 
increasing the $\mathrm{Nd}$ concentration in the intergranular phase, the phase changes from ferromagnetic to non-ferromagnetic, which increases the required field for the nucleation of reversed domain and strengthening pinning force against domain wall motion. The average $\mathrm{Nd}+\mathrm{Pr}$ concentrations in the $c$-plane intergranular phases of HDM-A and HDM-C were determined to be $\sim 50.1$ at.\% and $\sim 39.9$ at.\%, respectively, which are higher than the typical value for sintered magnets and comparable with that of the high coercivity hot-deformed magnets containing 14 at.\% $\mathrm{Nd}[7,29]$. The higher $\mathrm{Nd}+\mathrm{Pr}$ concentration in the $c$-planes of HDM-A compared to that of HDM-C indicates weaker intergrain exchange coupling along the $c$-axis of the HDM-A sample. However, the relatively low $\mathrm{Nd}+\mathrm{Pr}$ concentration is found in the $a b$-plane intergranular phases of HDM-C and HDM-A, which is considered as a barrier to increase the coercivity in hot-deformed magnets [8]. However, the $\mathrm{Nd}+\mathrm{Pr}$ content in the $a b$-plane intergranular phase was enhanced from $\sim 28.3$ at. $\%$ to $\sim 34.4$ at. $\%$ by processing hot-deformed magnets from amorphous precursors, which is supposed to arise from the decreasing aspect ratio and reduced gap of interfacial energy between the $a b$-plane and $c$-plane. In the typical hot-deformed magnets processed by the eutectic diffusion process, even though the concentration of $\mathrm{Nd}$ in the $c$-plane intergranular phases can be increased drastically, the $\mathrm{Nd}$ content in the $a b$-plane intergranular phases does not increase after the diffusion process [8]. Low $\mathrm{Nd}$ content of the intergranular phase in the $a b$-planes is therefore believed to impede further increase of coercivity [8]. Herein, we demonstrated that $\mathrm{Nd}+\mathrm{Pr}$ content in the $a b$-plane intergranular phases can be increased by processing hot-deformed magnets from amorphous precursors, which is also an important factor for the higher coercivity in HDM-A.

To discuss the influence of the aspect ratio of the $\mathrm{Nd}_{2} \mathrm{Fe}_{14} \mathrm{~B}$ grains on coercivity, $H_{\mathrm{c}} / M_{\mathrm{s}}$ values measured at different temperatures are plotted against the value of $H_{\mathrm{A}} / M_{\mathrm{s}}$ for the hot-deformed magnets produced from amorphous and crystalline precursors. Generally, the 
coercivity is phenomenologically expressed by $H_{\mathrm{c}}(T)=\alpha H_{\mathrm{A}}(T)-N_{\mathrm{eff}} M_{\mathrm{s}}(T)$; where $H_{\mathrm{A}}(\mathrm{T})$ and $M_{\mathrm{s}}(\mathrm{T})$ are the anisotropy field and saturation magnetization at different temperatures, $\alpha$ describes the reduced anisotropy field caused by defects at grain boundaries, and $N_{\text {eff }}$ is the effective demagnetizing factor [30-31], reflecting local demagnetization field. By measuring the temperature dependence of coercivity, $\alpha$ and $N_{\text {eff }}$ can be determined by plotting $H_{\mathrm{c}} / M_{\mathrm{s}}$ against $H_{\mathrm{A}} / M_{\mathrm{s}}$ at different temperatures and subsequent linear fitting the data. As shown in Fig. 10, $\alpha$ values were calculated to be 0.53 and 0.58 for HDM-C and HDM-A, respectively. Slightly higher $\alpha$ value for HDM-A is considered to be the result of the more continuous networking of grain boundary phases indicated in Fig. 5 and shrinking areal fraction of grain boundaries shown in Table $1 . N_{\text {eff }}$ slightly increases from 1.27 to 1.36 by processing hot deformed magnets from amorphous precursors. This result appears not to be consistent with the smaller aspect ratio of the grains in HDM-A. Note that the thickness of the intergranular phases in HDM-C and HDM-A in Table 2 is smaller than the exchange length of $\sim 1.9 \mathrm{~nm}$ [25], implying the grains in HDM-C and HDM-A are exchange coupled. This may nullify the contribution of smaller aspect ratio to reduce the local demagnetizing constant, $N_{\text {eff. }}$ On the other hand, the larger grain size in HDM-A results in larger $N_{\text {eff }}$ based on micromagnetic simulation [14]. As a result, $N_{\text {eff }}$ for HDM-A is slightly larger than that for HDM-C.

\section{Conclusion}

Hot-deformed magnets have been processed from amorphous and nanocrystalline precursors and their coercivities and microstructures have been compared to explore the optimum process route to maximize the coercivity. The hot-deformed magnets processed from an amorphous precursor exhibited a coercivity of $1.40 \mathrm{~T}$ that is higher than that processed 
from nanocrystalline powder, $\sim 1.28 \mathrm{~T}$. The average grain size of the hot-deformed magnets processed from amorphous precursor were larger than that processed from the nanocrystalline precursor, which caused higher $\mathrm{Nd}$ concentration in the intergranular phase, leading to stronger pinning force against reversed domain wall motion and higher coercivity.

\section{Acknowledgment}

This work was supported by JST, CREST. 


\section{References}

[1] J.J. Croat, J.F. Herbst, R.W. Lee, F.E. Pinkerton, High-energy product $\mathrm{Nd}-\mathrm{Fe}-\mathrm{B}$ permanent magnets, Appl. Phys. Lett. 44 (1984) 148-149.

[2] M. Sagawa, S. Fujimura, H. Yamamoto, Y. Matsuura, New material for permanent magnets on a base of Nd and Fe, J. Appl. Phys. 55 (1984) 2083-2087.

[3] M. Sagawa, S. Fujimura, H. Yamamoto, Y. Matsuura, K. Hiraga, Permanent magnet materials based on the rare earth-iron-boron tetragonal compounds, IEEE Trans. Magn. Mag. 20 (1984) 1584-1589.

[4] R.W. Lee, E.G. Brewer, N.A. Schaffel, Processing of Neodymium-Iron-Boron melt-spun ribbons to fully dense magnets, IEEE Trans. Magn. MAG-21 (1985) 1958-1963.

[5] R.W. Lee, Hot-pressed neodymium-iron-boron magnets, Appl. Phys. Lett. 46 (1985) 790-791.

[6] K. Hono, H. Sepehri-Amin, Strategy for high-coercivity Nd-Fe-B magnets, Scr. Mater. 67 (2012) 530-535.

[7] J. Liu, H. Sepehri-Amin, T. Ohkubo, K. Hioki, A. Hattori, T. Schrefl, K. Hono, Effect of $\mathrm{Nd}$ content on the microstructure and coercivity of hot-deformed $\mathrm{Nd}-\mathrm{Fe}-\mathrm{B}$ permanent magnets, Acta Mater. 61 (2013) 5387-5399.

[8] H. Sepehri-Amin, T. Ohkubo, S. Nagashima, M. Yano, T. Shoji, A. Kato, T. Schrefl, K. Hono, High-coercivity ultrafine-grained anisotropic $\mathrm{Nd}-\mathrm{Fe}-\mathrm{B}$ magnets processed by hot deformation and the $\mathrm{Nd}-\mathrm{Cu}$ grain boundary diffusion process, Acta Mater. 61 (2013) $6622-6634$.

[9] H. Sepehri-Amin, J. Liu, T. Ohkubo, K. Hioki, A. Hattori, K. Hono, Enhancement of coercivity of hot-deformed $\mathrm{Nd}-\mathrm{Fe}-\mathrm{B}$ anisotropic magnet by low-temperature grain boundary diffusion of $\mathrm{Nd}_{60} \mathrm{Dy}_{20} \mathrm{Cu}_{20}$ eutectic alloy, Scr. Mater. 69 (2013) 647-650. 
[10] T. Akiya, J. Liu, H. Sepehri-Amin, T. Ohkubo, K. Hioki, A. Hattori, K. Hono, Low temperature diffusion process using rare earth-Cu eutectic alloys for hot-deformed Nd-Fe-B bulk magnets, J. Appl. Phys. 115 (2014) 17A766-1-3.

[11] T.G. Woodcock, Q.M. Ramasse, G. Hrkac, T. Shoji, M. Yano, A. Kato, O. Gutfleisch, Atomic-scale features of phase boundaries in hot deformed $\mathrm{Nd}-\mathrm{Fe}-\mathrm{Co}-\mathrm{B}-\mathrm{Ga}$ magnets infiltrated with a Nd-Cu eutectic liquid, Acta Mater. 77 (2014) 111-124.

[12] T. Akiya, J. Liu, H. Sepehri-Amin, T. Ohkubo, K. Hioki, A. Hattori, K. Hono, High-coercivity hot-deformed $\mathrm{Nd}-\mathrm{Fe}-\mathrm{B}$ permanent magnets processed by $\mathrm{Nd}-\mathrm{Cu}$ eutectic diffusion under expansion constraint, Scr. Mater. 81 (2014) 48-51.

[13] L. Liu, H. Sepehri-Amin, T. Ohkubo, M. Yano, A. Kato, T. Shoji, K. Hono, Coercivity enhancement of hot-deformed Nd-Fe-B magnets by the eutectic grain boundary diffusion process, J. Alloy. Compd. 666 (2016) 432-439.

[14] J. Liu, H. Sepehri-Amin, T. Ohkubo, K. Hioki, A. Hattori, T. Schrefl, K. Hono, Grain size dependence of coercivity of hot-deformed $\mathrm{Nd}-\mathrm{Fe}-\mathrm{B}$ anisotropic magnets, Acta Mater. 82 (2015) 336-343.

[15] Y. Matsuura, J. Hoshijima, R. Ishii, Relation between $\mathrm{Nd}_{2} \mathrm{Fe}_{14} \mathrm{~B}$ grain alignment and coercive force decrease ratio in NdFeB sintered magnets, J. Magn. Magn. Mater. 336 (2013) $88-92$.

[16] J. Liu, H. Sepehri-Amin, T. Ohkubo, K. Hioki, A. Hattori, K. Hono, Microstructure evolution of hot-deformed Nd-Fe-B anisotropic magnets, J. Appl. Phys. 115 (2014) 17A744-1-3.

[17] H. Sepehri-Amin, W. F. Li, T. Ohkubo, T. Nishiuchi, S. Hirosawa, K. Hono, Effect of Ga addition on the microstructure and magnetic properties of hydrogenation-disproportionationdesorption-recombination processed Nd-Fe-B powder, Acta Mater. 58 (2010) 1309. 
[18] I. Ahmad, H. A. Davies, R. A. Buckley, Ultra high coercivity Nd-Fe-B permanent magnet alloy with small addition of Ga, Mater. Lett. 20 (1994) 139-142.

[19] T.T. Sasaki, T. Ohkubo, Y. Takada, T. Sato, A. Kato, Y. Kaneko, K. Hono, Formation of non-ferromagnetic grain boundary phase in a Ga-doped Nd-rich $\mathrm{Nd}-\mathrm{Fe}-\mathrm{B}$ sintered magnet, Scr. Mater. 113 (2016) 218-221.

[20] T. T. Sasaki, T. Ohkubo, and K. Hono, Structure and chemical compositions of the grain boundary phase in Nd-Fe-B sintered magnets, Acta Mater. 115 (2016) 269-277.

[21] T.T. Sasaki, T. Ohkubo, Y. Une, H. Kubo, M. Sagawa, K. Hono, Effect of carbon on the coercivity and microstructure in fine-grained Nd-Fe-B sintered magnet, Acta Mater. 84 (2015) 506-514.

[22] R. Harris, M. Plischke, M. J. Zuckermann, New model for amorphous magnetism, Phys. Rev. Lett. 31 (1973) 160-162.

[23] E. Callen, Y. J. Liu, J. R. Cullen, Initial magnetization, remanence, and coercivity of the random anisotropy amorphous ferromagnet, Phys. Rev.B 16 (1977) 263-270.

[24] R. Alben, J.J. Becker, M.C. Chi, Random anisotropy in amorphous ferromagnets, J. Appl. Phys. 49 (1978) 1653-1658.

[25]J.M.D. Coey, Magnetism and magnetic materials, Cambridge University Press, Cambridge, 2010.

[26] W. Li, L.L. Li, Y. Nan, Z.Y. Xu, X.Y. Zhang, A.G. Popov, D.V. Gunderov, V.V. Stolyarov, Nanocrystallization and magnetic properties of amorphous $\mathrm{Nd}_{9} \mathrm{Fe}_{85} \mathrm{~B}_{6}$ subjected to high-pressure torsion deformation upon annealing, J. Appl. Phys. 104 (2008) 023912-1-7.

[27] A. Manaf, R. A. Buckley, H. A. Davies, and M. Leonowicz, Enhanced magnetic properties in rapidly solidified Nd-Fe-B based alloys, J. Magn. Magn. Mater. 101 (1991) 360-362. 
[28] T. Schrefl, J. Fidler, H. Kronmüller, Remanence and coercivity in isotropic nanocrystalline permanent magnets, Phys. Rev. B 49 (1994) 6100-6110.

[29] W.F. Li, T. Ohkubo, K. Hono, Effect of post-sinter annealing on the coercivity and microstructure of Nd-Fe-B permanent magnets, Acta Mater. 57 (2009) 1337-1346.

[30] H. Kronmüller, The nucleation fields of uniaxial ferromagnetic crystals, Phys. Status Solidi B 130 (1985) 197-203.

[31] S. Hock, PhD thesis, Max-Planck-Institute for Metals Research, Institute of Physics, 1988, p. 118. 


\section{Tables}

Table 1 Grain size, aspect ratio (AR) of grains and areal fraction of grain boundary (AFGB) phases in hot-deformed magnets (HDMs)

\begin{tabular}{ccccc}
\hline HDMs & $D_{\mathrm{c}}$ & $D_{\mathrm{ab}}$ & AR of Grains & AFGB phases \\
\hline HDM-C & $375 \mathrm{~nm}$ & $106 \mathrm{~nm}$ & 3.54 & $2.58 \%$ \\
HDM-A & $343 \mathrm{~nm}$ & $132 \mathrm{~nm}$ & 2.60 & $2.27 \%$ \\
\hline
\end{tabular}

Table 2 Average concentration and thickness $(d)$ of $c$-plane and side-plane grain boundary (GB) phases in hot-deformed magnets (HDMs)

\begin{tabular}{lccccccc}
\hline \multirow{2}{*}{ HDMs } & \multicolumn{3}{c}{$c$-Plane GB phases } & & \multicolumn{3}{c}{ side-Plane GB phases } \\
\cline { 2 - 4 } \cline { 6 - 8 } & $\mathrm{Nd}+\mathrm{Pr}$ & $\mathrm{Fe}+\mathrm{Co}$ & $d$ & & $\mathrm{Nd}+\mathrm{Pr}$ & $\mathrm{Fe}+\mathrm{Co}$ & $d$ \\
\hline HDM-C & 39.9 at. $\%$ & 51.2 at. $\%$ & $0.9 \mathrm{~nm}$ & & 28.3 at. $\%$ & 64.4 at. $\%$ & $0.88 \mathrm{~nm}$ \\
HDM-A & 50.1 at. $\%$ & 40.2 at. $\%$ & $1.21 \mathrm{~nm}$ & & 34.4 at. $\%$ & 57.9 at. $\%$ & $1.07 \mathrm{~nm}$ \\
\hline
\end{tabular}




\section{Figures' Captions}

Fig. 1. XRD patterns for magnetic powders, hot-pressed and hot-deformed magnets.

Fig. 2. Hysteresis loops for magnetic powders (a) and demagnetization curves for hot-pressed and hot-deformed magnets (b).

Fig. 3. Bright field TEM images and inset SADPs of (a) nanocrystalline powders and (b) amorphous powders.

Fig. 4. Bright field TEM image and inset SADP for (a) HPM-C and (b) HPM-A, pressing direction is indicated by red arrow denoted as P; high-resolution HAADF-STEM images and superimposed EDS mappings of typical Nd-rich grain boundary (GB) phases in (c) HPM-C and (d) HPM-A, GB phase is indicated by red arrow and lattice fringes diffused area is marked by red dashed circle; (e) and (f) compositional profiles across the Nd-rich grain boundary phase in (c) and (d), respectively.

Fig. 5. Low magnification cross-sectional BSE SEM images of (a) HDM-C and (b) HDM-A; high magnification cross-sectional BSE SEM images of (c) HDM-C and (d) HDM-A. pressing direction denoted as $\mathrm{P}$ is shown by arrow and $c$ axis is in-plane as the arrow indicates.

Fig. 6. HAADF image (a) and STEM-EDS elemental mappings of HDM-C for (b) $\mathrm{Nd}$, (c) Fe and (d) Ga from the same region as (a).

Fig. 7. HAADF image (a) and STEM-EDS elemental mappings of HDM-A for (b) $\mathrm{Nd}$, (c) Fe and (d) Ga from the same region as (a).

Fig. 8. High-resolution HAADF-STEM images and superimposed EDS mappings of typical Nd-rich grain boundary phases in $c$-plane of HDM-C (a-c) and HDM-A (j-1); (d-f) and (g-h) compositional profiles across the Nd-rich grain boundary phase in (a-c) and (j-1), respectively. 
Fig. 9. High-resolution HAADF-STEM images and superimposed EDS mappings of typical Nd-rich grain boundary phases in side-plane of HDM-C (a-c) and HDM-A (j-1); (d-f) and (g-h) compositional profiles across the Nd-rich grain boundary phase in (a-c) and (j-1), respectively.

Fig. 10. Dependence of $H_{\mathrm{c}} / M_{\mathrm{s}}$ on $H_{\mathrm{a}} / M_{\mathrm{s}}$ of the HDM-C and HDM-A samples. Micromagnetic parameters $\alpha$ and $N_{\text {eff }}$ are calculated from the slope and intercept of the linear fit line of these plots, respectively. 


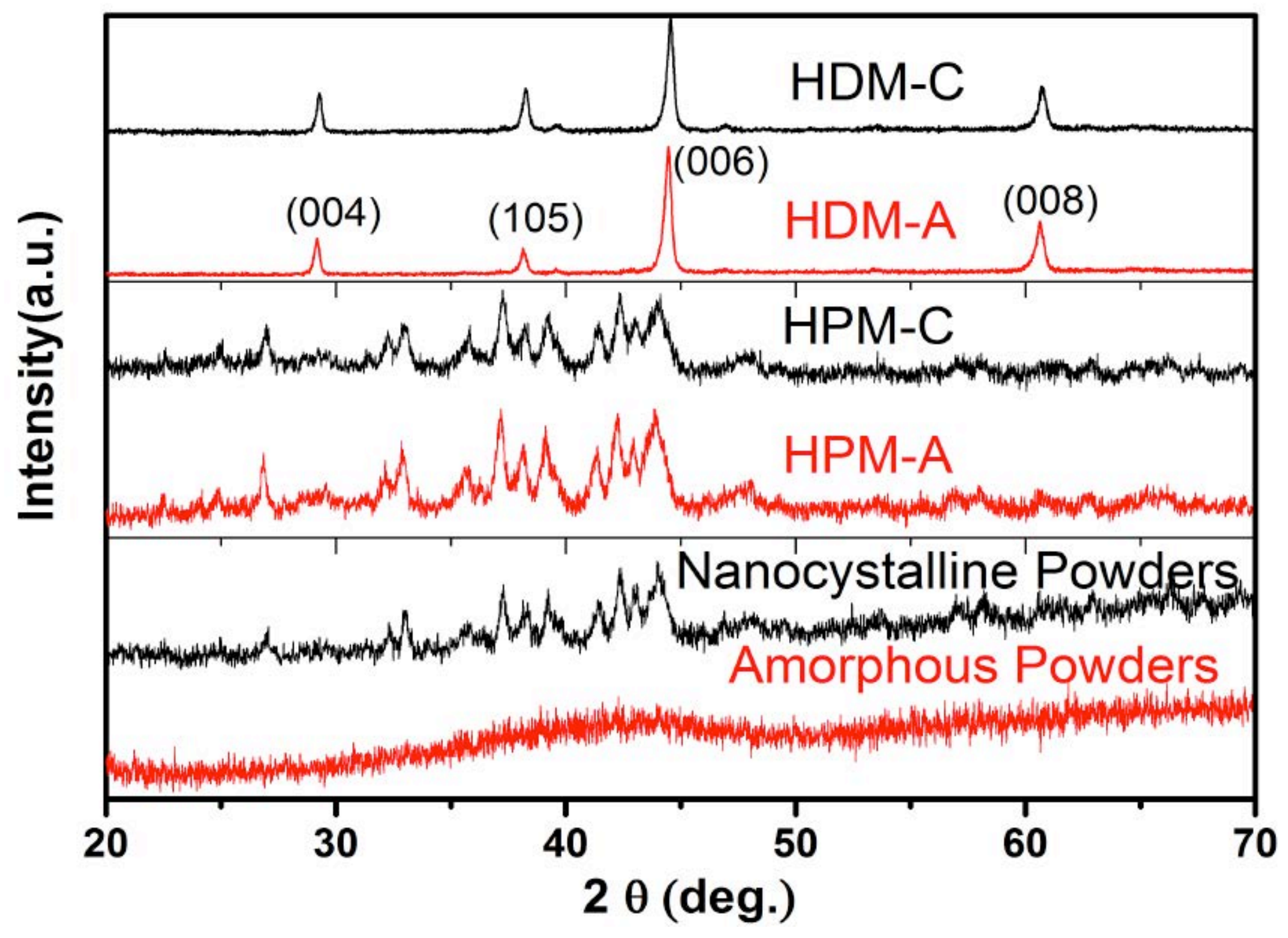

FIGURE 1 

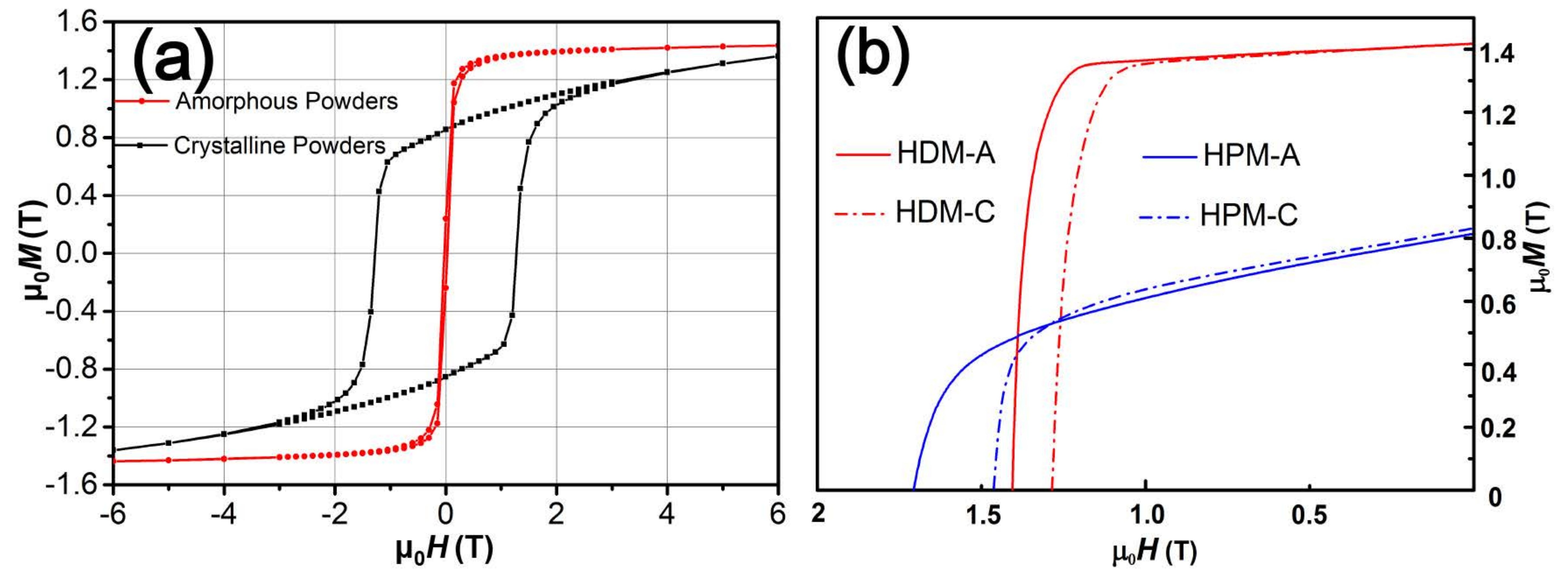


\section{FIGURE 3}

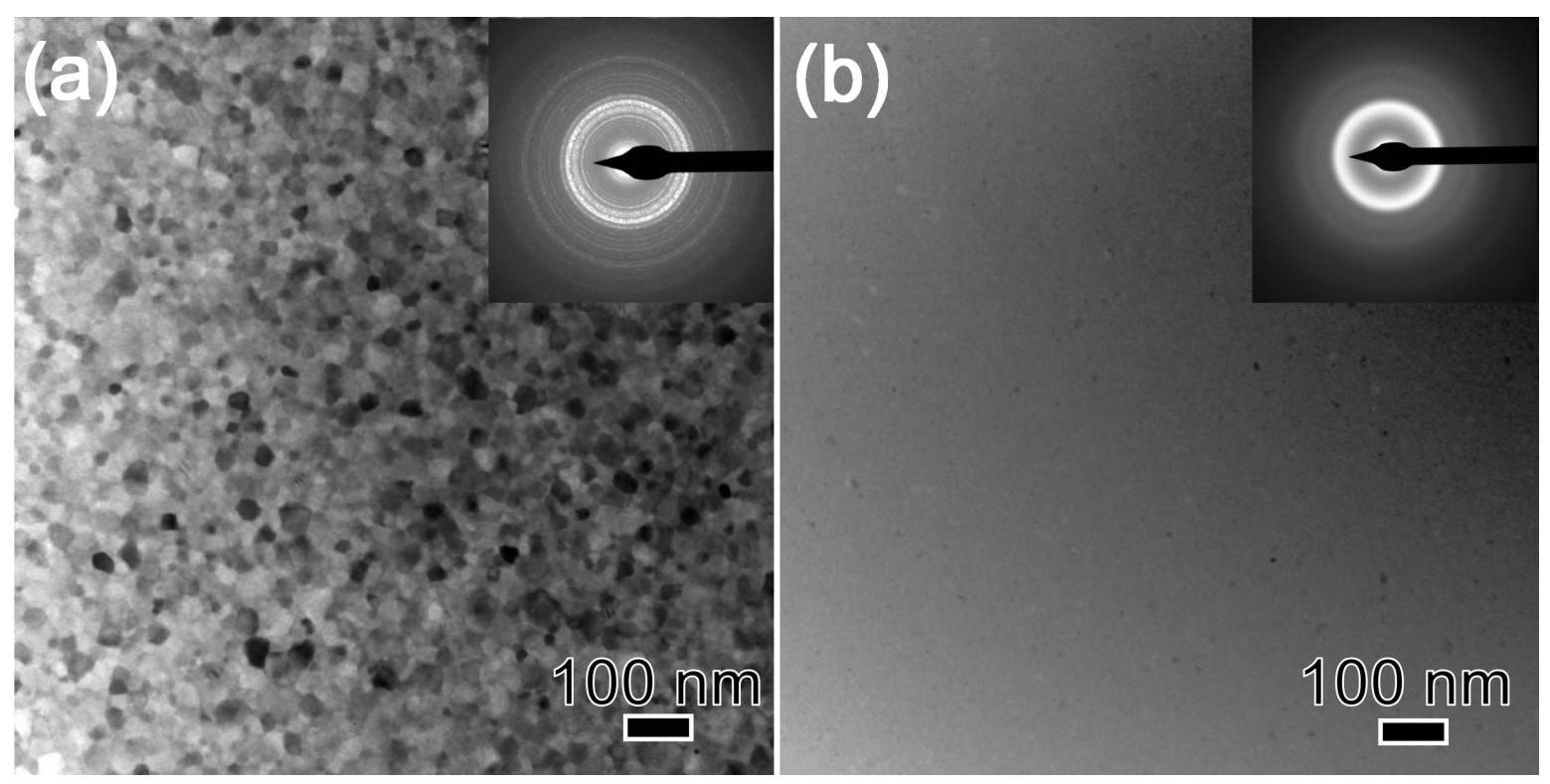




\section{FIGURE 4}
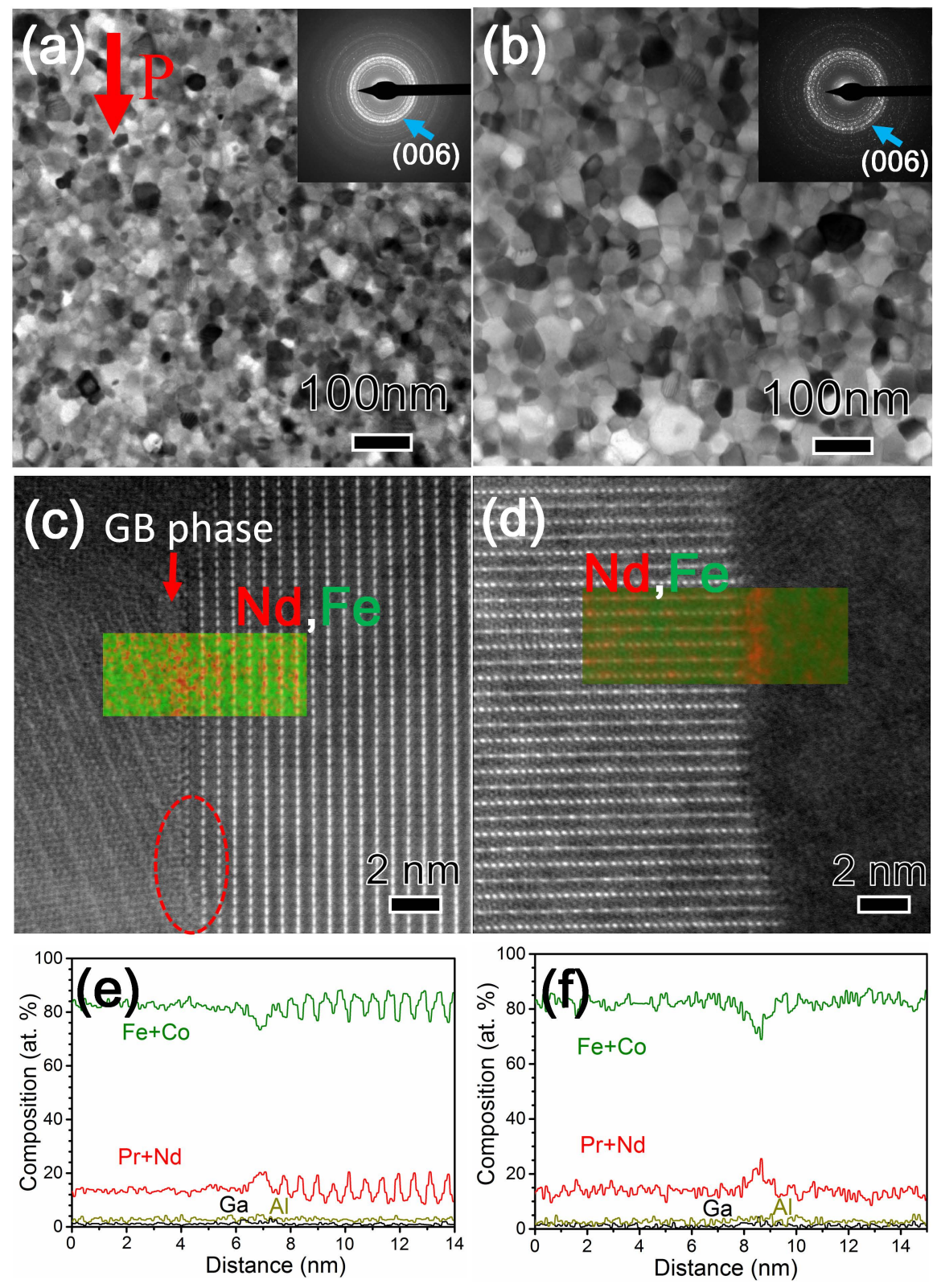
FIGURE 5

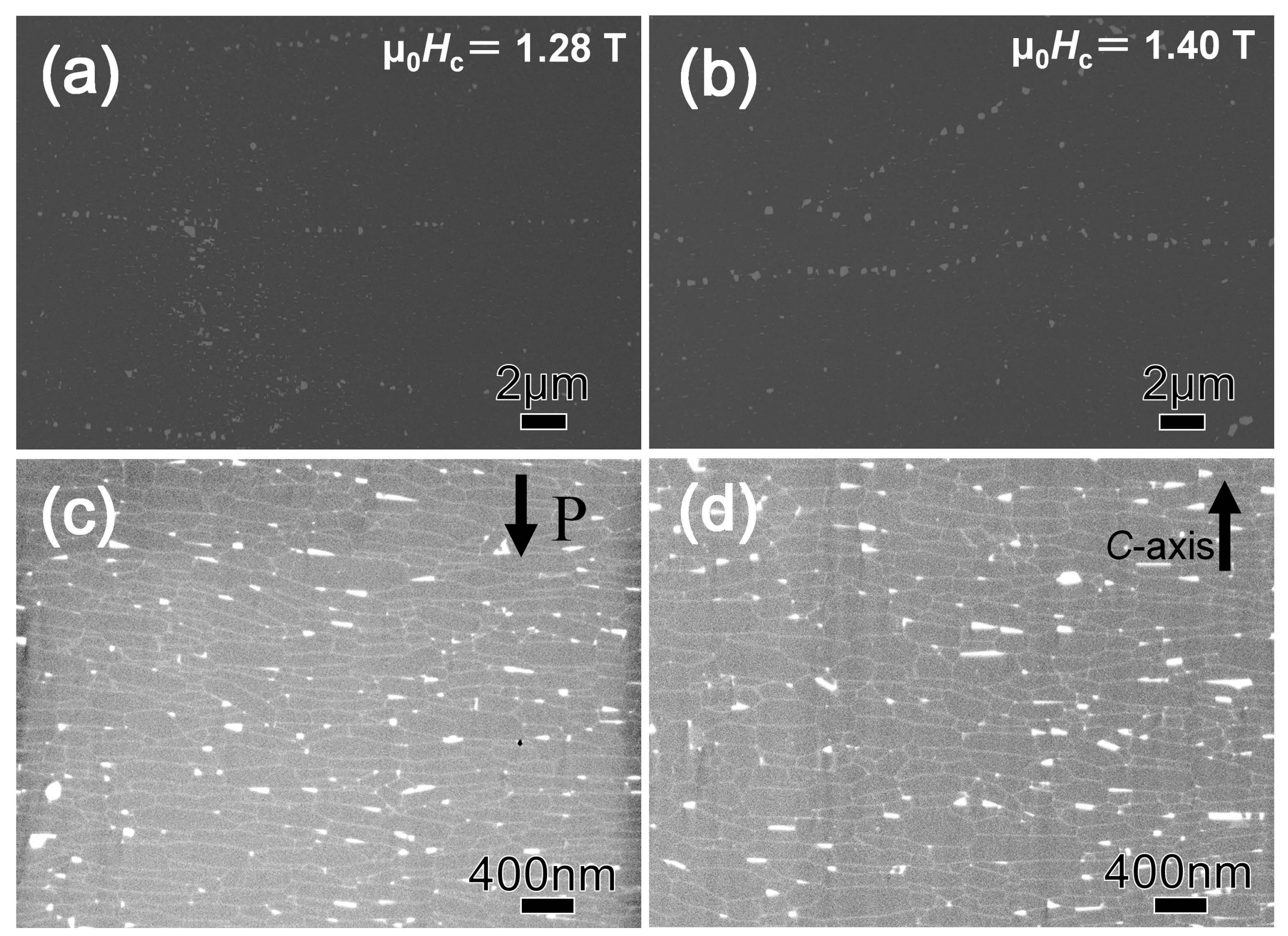




\section{FIGURE 6}

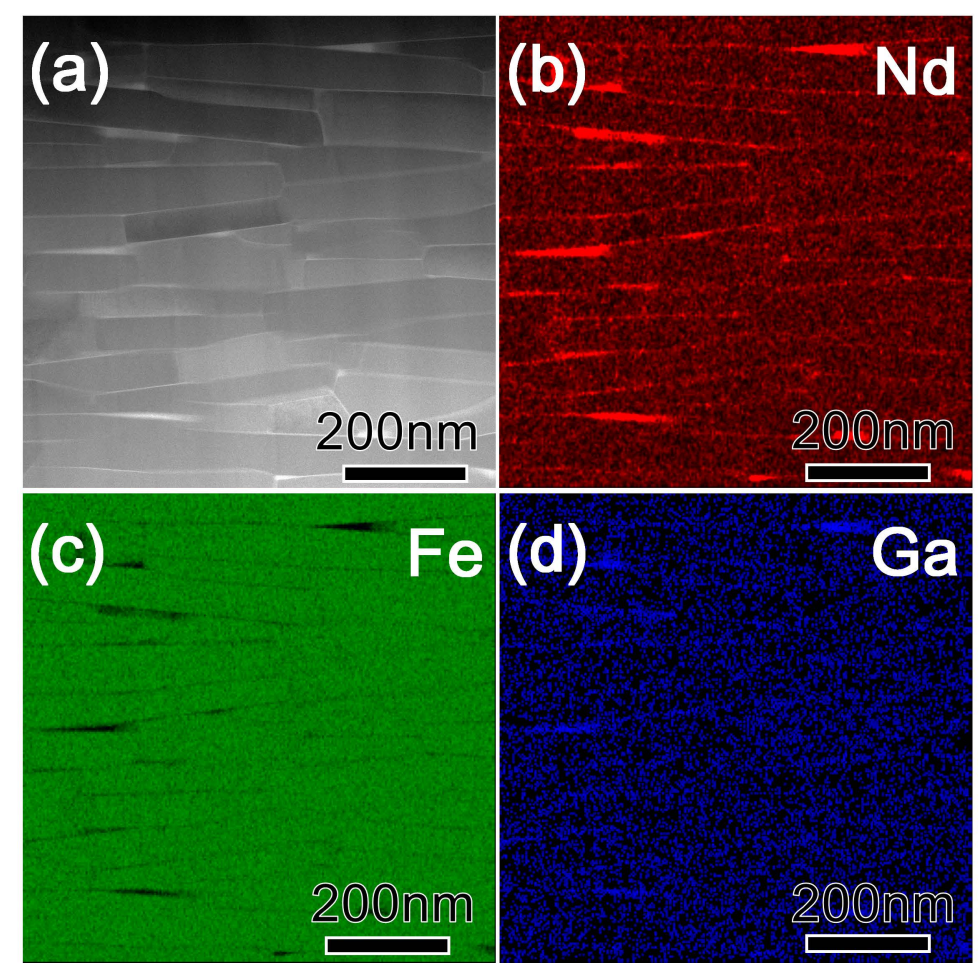




\section{FIGURE 7}

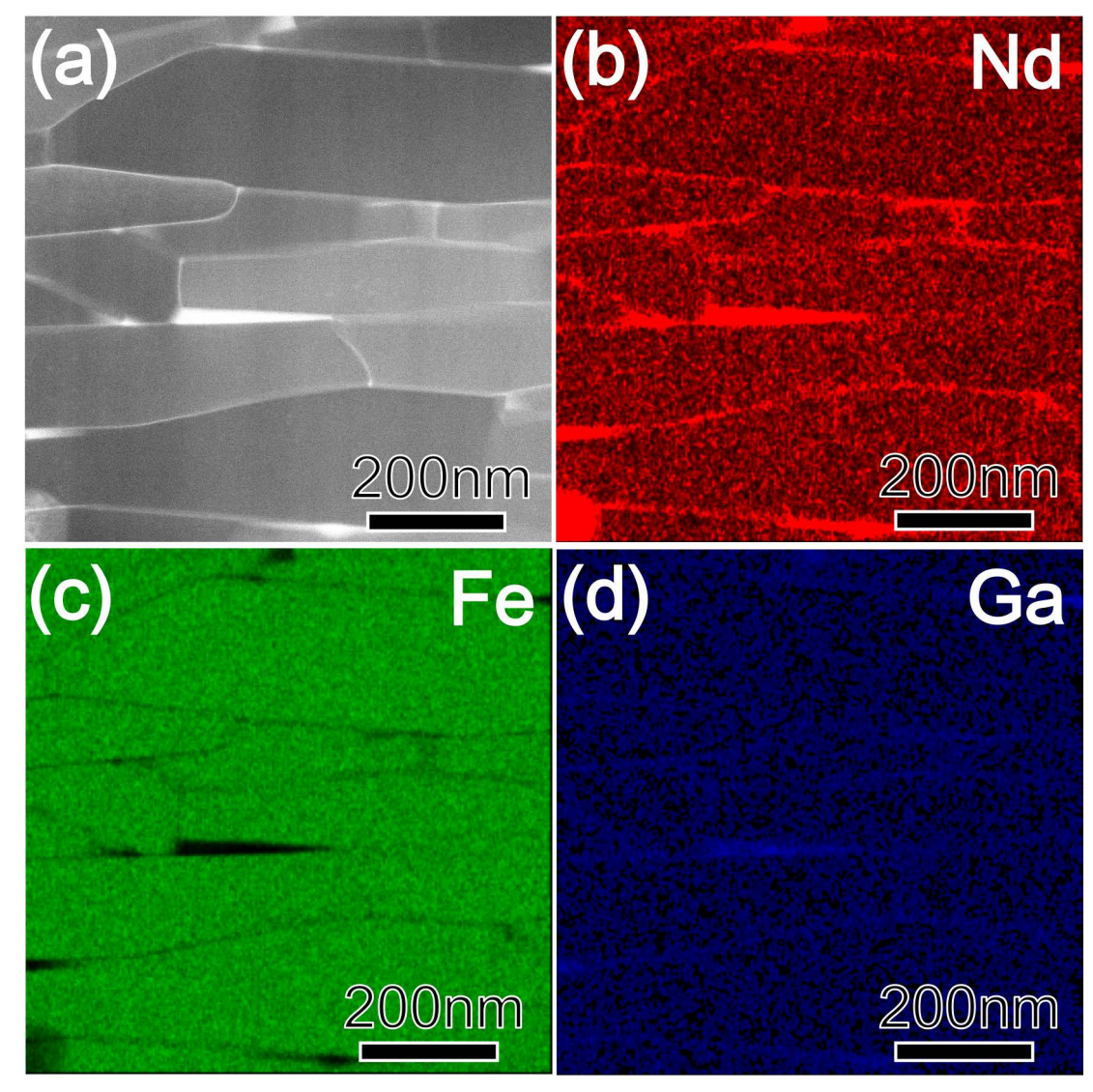


FIGURE 8
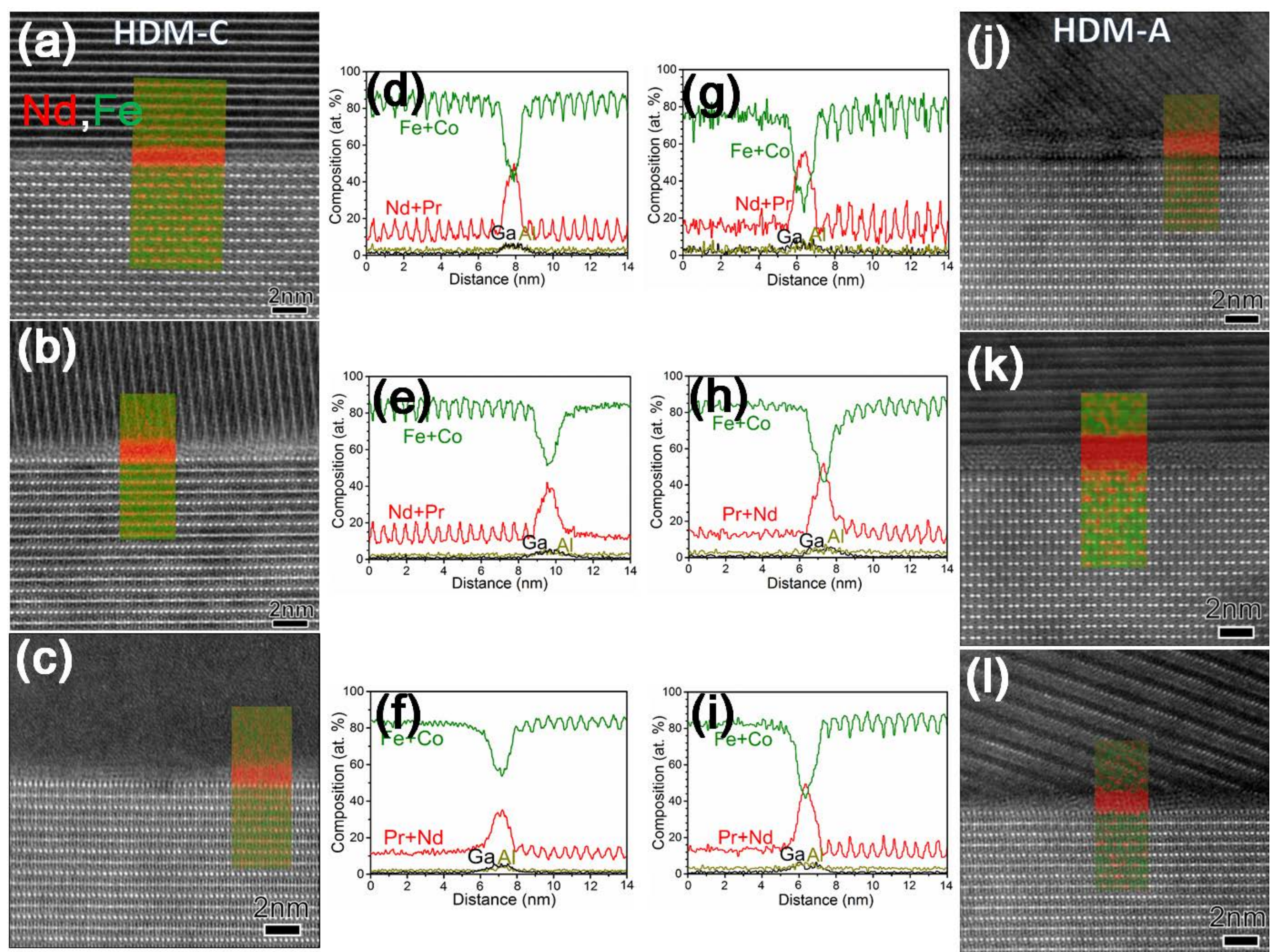

(I)

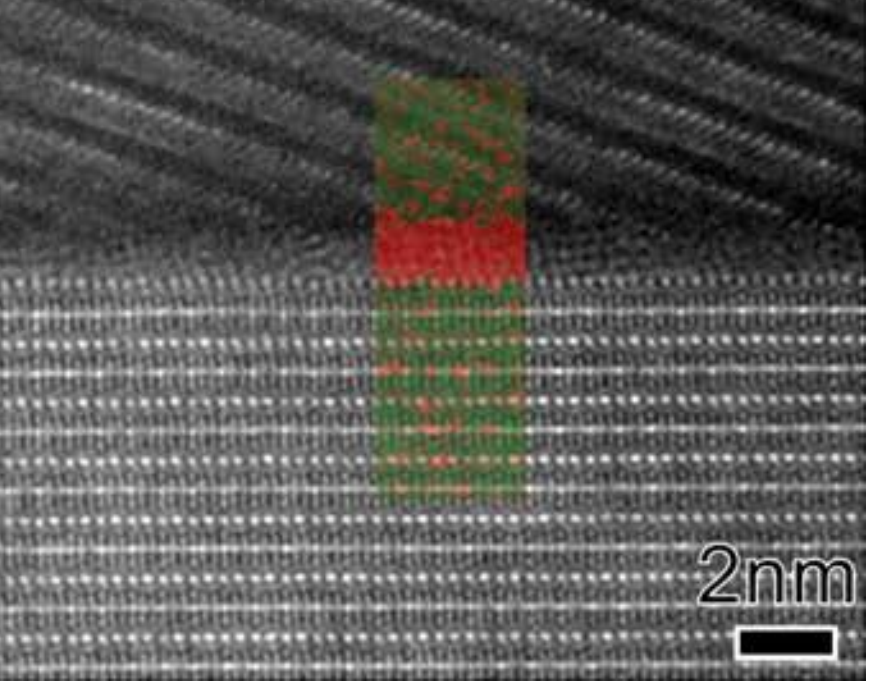


FIGURE 9
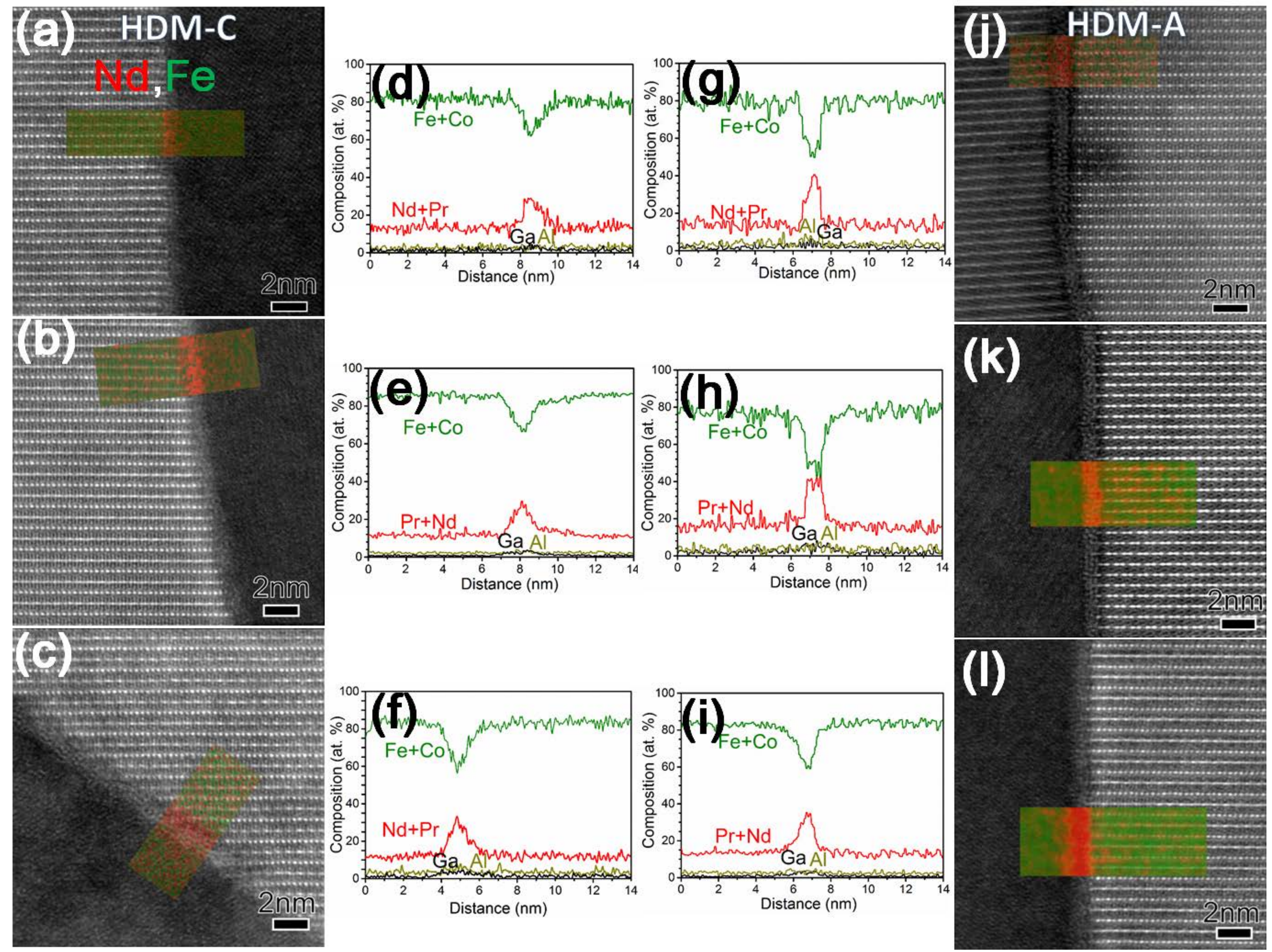
FIGURE 10

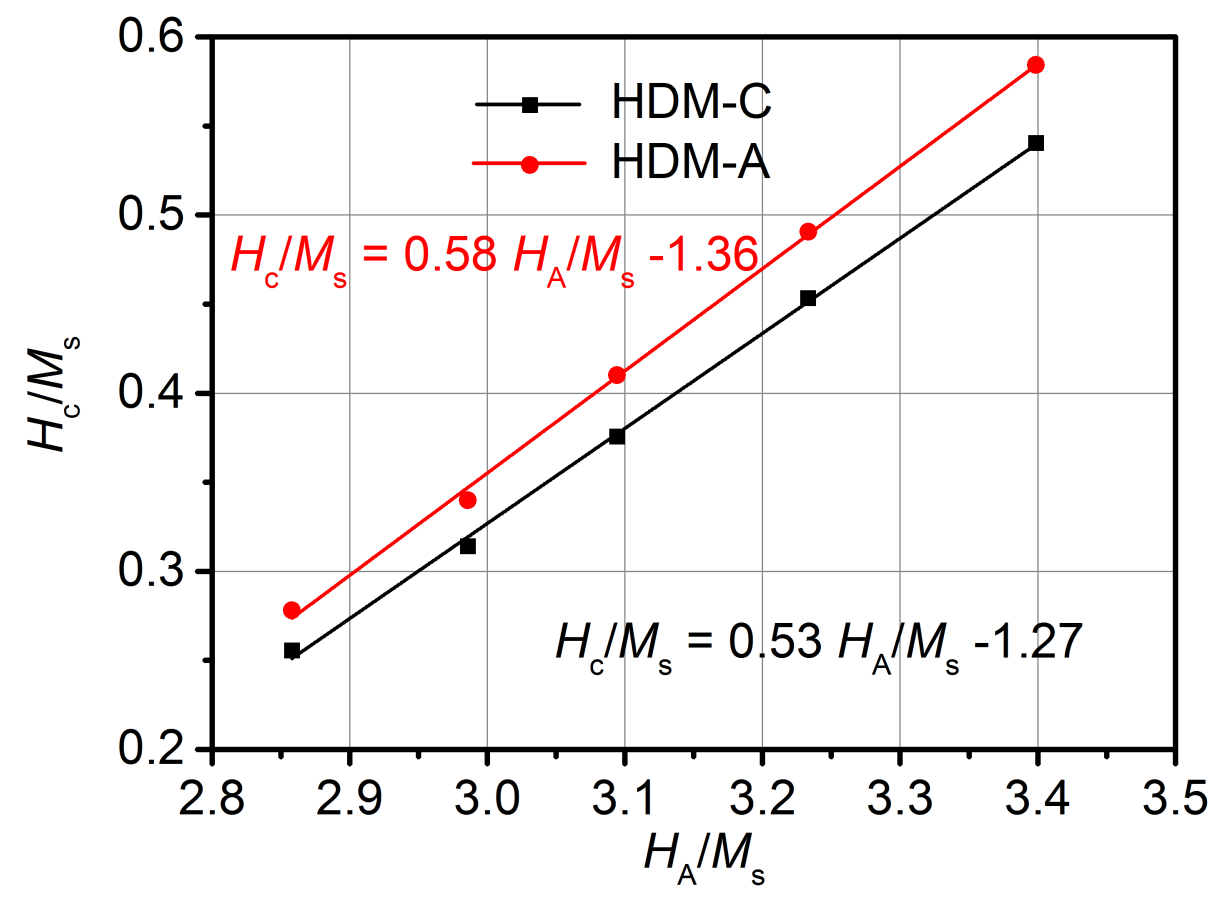




\section{*Graphical Abstract}

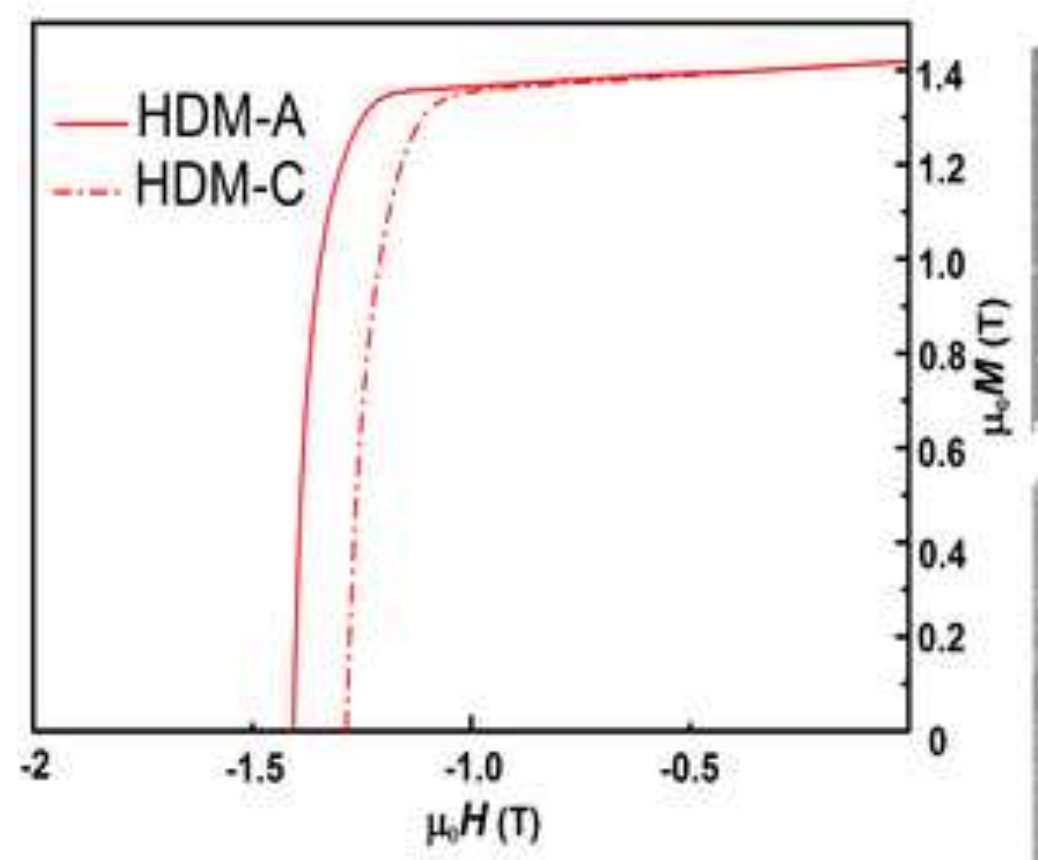

Hot deformed magnets from crystalline ribbons (HDM-C)
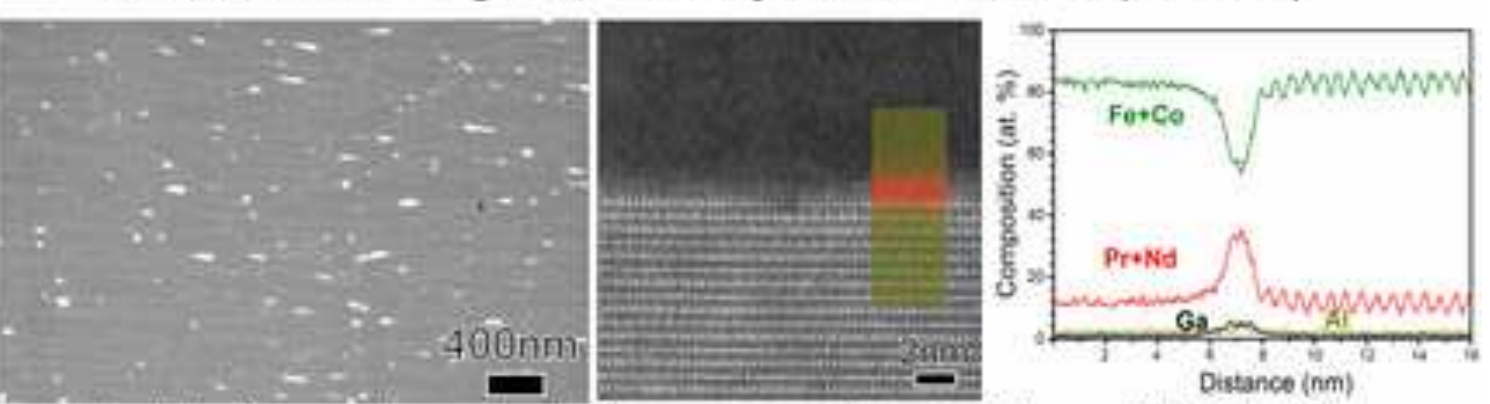

Hot deformed magnets from amorphous ribbons (HDM-A)
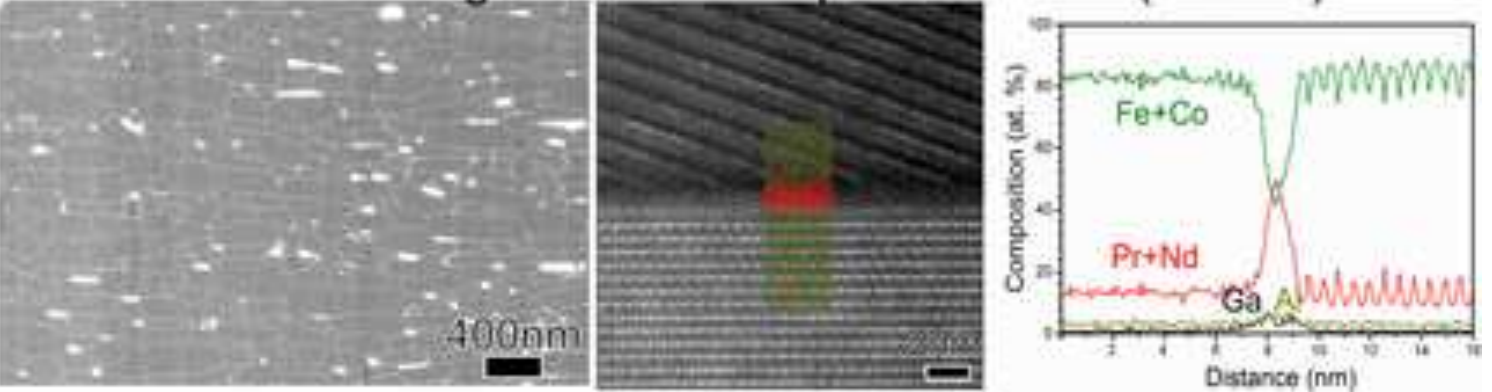